\title{
Functional redundancy of the Notch pathway in ovarian cancer cell lines
}

\author{
FERNANDA SILVA $^{1,2}$, ANA FÉLIX ${ }^{1-3}$ and JACINTA SERPA ${ }^{1,2}$ \\ ${ }^{1}$ Chronic Diseases Research Center (CEDOC-FCM-UNL), NOVA Medical School, NOVA University of Lisbon, \\ Lisbon 1169-056; ${ }^{2}$ Molecular Pathobiology Research Unit; ${ }^{3}$ Department of Pathology, \\ Portuguese Institute of Oncology Francisco Gentil (IPOLFG), Lisbon 1099-023, Portugal
}

Received July 17, 2015; Accepted April 29, 2016

DOI: $10.3892 / \mathrm{ol} .2016 .4959$

\begin{abstract}
Epithelial ovarian cancer is the most lethal gynecologic malignancy, despite advances in treatment. The most common histological type, high-grade ovarian serous carcinoma (OSC) is usually diagnosed at an advanced stage, and although these types of tumors frequently respond to surgery and platinum-based chemotherapy, they usually recur. Ovarian clear cell carcinoma (OCCC) is an unusual histological type, which is known to be intrinsically chemoresistant and is associated with poor prognosis in advanced stages. In recent years, genetic alterations and epigenetic modulation of signaling pathways have been reported in OSC and OCCC, including the overexpression of Notch pathway elements and histone deacetylases. Histone deacetylase inhibitors (HDACis), including vorinostat (suberoylanilide hydroxamic acid), alter the transcription of genes involved in cell growth, survival and apoptosis, and have become an attractive therapeutic approach. However, no previous work has addressed the effect of HDACis, and in particular vorinostat, on Notch signaling in ovarian cancer. Therefore, the present study aimed to investigate the modulation of the Notch pathway by vorinostat in ovarian cancer. Using immunofluorescence and quantitative polymerase chain reaction, the present results revealed that vorinostat activated the Notch pathway in OCCC and OSC cell lines, through different Notch ligands. In OCCC, the activation of the Notch pathway appeared to occur through Delta-like (D1l) ligands 1, 2 and 3, whereas in OSC D111 and Jagged 1 and 2 ligands were involved. The activation of the Notch pathway by vorinostat, in OCCC and OSC cell lines, culminated in the increased expression of the same downstream transcription factors, hairy enhancer of split (Hes) 1 and 5, and Hes-related proteins 1 and 2. In conclusion, vorinostat
\end{abstract}

Correspondence to: Dr Fernanda Silva, Chronic Diseases Research Center (CEDOC-FCM-UNL), NOVA Medical School, NOVA University of Lisbon, Campo Mártires da Pátria 130, Lisbon 1169-056, Portugal

E-mail: fernanda.silva@fcm.unl.pt

Key words: ovarian cancer, Notch pathway, vorinostat modulates the expression of several downstream targets of the Notch pathway and independent Notch receptors and ligands that are expressed in OSC and OCCC. This upregulation of the Notch pathway may explain why vorinostat therapy fails in ovarian carcinoma treatment, as shown in certain clinical trials.

\section{Introduction}

Epithelial ovarian cancer (EOC) is the most lethal and the seventh most common gynecologic malignancy in women worldwide $(1,2)$. EOC represents $90 \%$ of ovarian malignant tumors (3) and is an extremely heterogeneous group of neoplasms that exhibit a wide range of tumor morphology, clinical manifestations and underlying genetic alterations (4). The most common histological type, high-grade ovarian serous carcinoma (OSC), is characterized by tumor protein p53 mutations and breast cancer 1 and 2 dysfunction (5). This type of tumor is aggressive and usually diagnosed at an advanced stage, and although OSC frequently responds to surgery and platinum-based chemotherapy, it usually recurs (6). Ovarian clear cell carcinoma (OCCC) is a more unusual histotype of EOC, which is known to be intrinsically chemoresistant and is associated with poor prognosis in advanced stages (6). Molecular alterations of OCCC are not well known, presenting a challenge to treat this type of tumor. However, OCCC is characterized by a unique histology, de novo expression of hepatocyte nuclear factor- $1 \beta$ transcription factor and somatic mutations of AT-rich interaction domain 1A gene, and loss of expression $(7,8)$. In previous years, other genetic alterations and epigenetic modulation of signaling pathways have been reported in OSC and OCCC (9), including the overexpression of Notch pathway elements and histone deacetylases (HDAC) (10). The Notch pathway has multiple roles in cell fate determination, since it regulates cell proliferation, differentiation, survival and apoptosis $(11,12)$. This signaling pathway is deregulated in human hematological malignancies and solid tumors $(13,14)$, and it is also implicated in angiogenesis $(15,16)$. Notch signaling is a juxtacrine pathway composed by Notch receptors (Notch1-4) and two classes of ligands, Delta-like (DIl) 1, 3 and 4 and serrate-like Jagged 1 and 2 (17-20).

Notch signaling is initiated by the binding of Delta/Jagged ligands to Notch receptors. Through several proteolytic 
cleavages, the Notch intracellular domain (NICD) is released and activates the transcription of target genes, hairy enhancer of split (Hes) family proteins, Hes-related proteins (Hey) (21) as well as cell cycle regulators, including $p 21^{\text {cipl/wafl }}(11)$, cyclin Dl and 3 (22), c-myc (23) and human epidermal growth factor 2 (24).

Epigenetic alterations are also involved in the repression of tumor suppressor genes and promotion of tumorigenesis in ovarian cancers, and HDAC inhibitor (HDACi) drugs are an attractive therapeutic approach (25). HDACis inhibit cancer cell growth in vitro and in vivo, revert oncogene-transformed cell morphology, induce apoptosis and enhance cell differentiation (26). Vorinostat (suberoylanilide hydroxamic acid) is a HDACi (27) that was FDA approved in 2006 for the treatment of cutaneous T-cell lymphoma, and it has demonstrated interesting results in in vitro models of ovarian cancer (28). However, to the best of our knowledge, there has been no previous study addressing the effect of HDACi, and in particular of vorinostat, on Notch signaling in ovarian cancer. Therefore, the aim of the present study was to investigate the modulation of the Notch pathway by vorinostat in ovarian cancer cell lines.

\section{Materials and methods}

Cell lines and cell culture conditions. OCCC ES2 (CRL-1978) and OSC OVCAR3 (HTB-161) cell lines were obtained from American Type Culture Collection (Manassas, VA, USA). The cells were incubated at $37^{\circ} \mathrm{C}$ in a humidified atmosphere containing 5\% $\mathrm{CO}_{2}$ in McCoy's 5A Modified Medium (Sigma-Aldrich, St. Louis, MO, USA) supplemented with $10 \%$ fetal bovine serum (FBS) and 1\% antibiotic-antimycotic (AA) (Invitrogen $^{\mathrm{TM}}$; Thermo Fisher Scientific, Inc., Waltham, MA, USA). The cells were cultured to $80-100 \%$ confluence prior to detachment by incubation with $1 \mathrm{X} 0.05 \%$ trypsin-EDTA (Invitrogen $^{\mathrm{TM}}$; Thermo Fisher Scientific, Inc.) at room temperature. For the various assays, cell number was determined using a Bürker counting chamber. Vorinostat (catalog no., CAS 149647-78-9; Cayman Chemical, Ann Arbor, MI, USA) was used at $5 \mu \mathrm{M}$ to treat cells.

Immunofluorescence. Cells were cultured on glass slides with a $0.2 \%$ gelatin coating in McCoy's 5A Modified Medium supplemented with $10 \%$ FBS, $1 \%$ AA until $80 \%$ confluence, and were then fixed in $2 \%$ paraformaldehyde for $15 \mathrm{~min}$ at $4^{\circ} \mathrm{C}$. Blocking was performed with $0.2 \%(\mathrm{w} / \mathrm{v})$ bovine serum albumin (BSA; catalog no., A9647; Sigma-Aldrich) in $1 \mathrm{X}$ phosphate-buffered saline (PBS) for $1 \mathrm{~h}$ at room temperature, and incubated with primary antibodies at $4^{\circ} \mathrm{C}$ overnight [dilution, 1:100 in $0.2 \%$ (w/v) BSA in $1 \mathrm{X}$ PBS]. The primary antibodies were as follows: Rabbit polyclonal anti-human Notch1 extracellular (catalog no., ABS90; EMD Millipore, Billerica, MA, USA), rabbit monoclonal anti-Notch1 cleaved (catalog no., 4147; Cell Signaling Technology, Inc., Danvers, MA, USA), rabbit polyclonal anti-Notch2 cleaved (catalog no., 07-1234; EMD Millipore) and rabbit polyclonal anti-Notch4 (catalog no., N5163; Sigma-Aldrich). The cells were incubated with Alexa Fluor ${ }^{\circledR} 488$ goat anti-rabbit secondary antibody (catalog no., A-11008; Invitrogen ${ }^{\mathrm{TM}}$, Thermo Fisher Scientific, Inc.) for $2 \mathrm{~h}$ at room temperature. The slides were mounted in VECTASHIELD Antifade Mounting Medium with DAPI (catalog no., H-1200; Vector Laboratories, Inc., Burlingame, CA, USA) and examined by standard fluorescence microscopy using an Axio Imager microscope (Zeiss GmbH, Jena, Germany). Images were acquired with AxioVision software (version 4.5; Zeiss $\mathrm{GmbH}$ ) and processed with ImageJ software (version $1.44 \mathrm{p}$; imagej.nih.gov/ij/).

Reverse transcription-quantitative polymerase chain reaction ( $q$ PCR). Total RNA was isolated from cells cultured in complete McCoy's with or without (control conditions) vorinostat, using RNeasy Mini Extraction kit (catalog no., 74104, Qiagen, Inc., Valencia, CA, USA), according to the manufacturer's protocol. cDNA synthesis was performed with $1 \mu \mathrm{g}$ total RNA, using random hexamers (catalog no., 11034731001; Roche Diagnostics, Indianapolis, IN, USA) and SuperScript II ${ }^{\mathrm{TM}}$ (200 U; Invitrogen $^{\mathrm{TM}}$, Thermo Fisher Scientific, Inc.), according to the manufacturer's protocol. The PCR amplification conditions were as follows: $95^{\circ} \mathrm{C}$ for $2 \mathrm{~min}, 95^{\circ} \mathrm{C}$ for $10 \mathrm{~min}$, followed by 45 cycles of $95^{\circ} \mathrm{C}$ for $15 \mathrm{sec}$ and $60^{\circ} \mathrm{C}$ for $1 \mathrm{~min}$. Dissociation curve conditions were as follows: $95^{\circ} \mathrm{C}$ for $15 \mathrm{sec}$ and $60^{\circ} \mathrm{C}$ for 15 sec. qPCR was performed using an ABI PRISM 7900HT Sequence Detection System (Applied Biosystems ${ }^{\circledR}$; Thermo Fisher Scientific, Inc.) with Power SYBR Green PCR Master Mix (catalog no., 4367659; Applied Biosystems ${ }^{\circledR}$, Thermo Fisher Scientific, Inc.). The primers sequences used were as follows: Notch1, TGGCGGGAAGTGTGAAGCGG (forward) and GTGCGAGGCACGGGTTGGG (reverse); Notch2, CCA TATGCTTCAGCCGGGATAC (forward) and GTCTCACAT TTCTGCCCTGTG (reverse); Notch3, CTGCAAGGACCG AGTCAATGG (forward) and CGTCCACGTTGCGATCAC AC (reverse); Nocth4, CCACCTTTCACCTCTGCCTC (forward) and ACCTCACAGTCTGGGCCTAT (reverse); Dll1, ATGCCTTCGGCCACTTCAC (forward) and CACATCCAG GCAGGCAGAT (reverse); D113, GAACCCGTGTGCCAA TGGAG (forward) and GTAGGCAGAGTAGGGTCTG (reverse); Dll4, GTGGGTCAGAACTGGTTATTGGA (forward) and TGACAGATGACCCGGTAAGAGT (reverse); Jagged1, CGGCTTTGCCATGTGCTT (forward) and TCT TCCTCCATCCCTCTGTCA (reverse); Jagged2, GTCGTC ATCCCCTTCCAGTTC (forward) and CTCATTCGGGGT GGTATCGTT (reverse); Hey1, GAAGTTGCGCGTTATCTG AG (forward) and GTTGAGATGCGAAACCAGTC (reverse); Hey2, TCGCCTCTCCACAATTCAG (forward) and TGA ATCCGCATGGGCAAACG (reverse); Hes1, CGGAGGTGC TTCACTGTCAT (forward) and ACGACACCGGATAAA CCAAA (reverse); Hes5, GAGAAAAACCGACTGCGGAAG (forward) and GACAGCCATCTCCAGGATGTC (reverse); Hes6, GAAGTGCTGGAGCTGACGG (forward) and CGA GCAGATGGTTCAGGAGC (reverse). All samples were run in triplicate. Data were analyzed in SDS 2.4.1 software (Applied Biosystems; Thermo Fisher Scientific, Inc.) and the relative expression of each gene was quantified by comparative quantification cycle $(\mathrm{Cq})$ method $(\Delta \Delta \mathrm{Ct})(29)$ using hypoxanthine phosphoribosyltransferase gene (HPRT) as an endogenous reference gene.

Statistical analysis. Statistical analysis was performed using Student's t-test with GraphPad Prism software (version 5.03; GraphPad Software, Inc., La Jolla, CA, USA). P<0.05 was considered to indicate a statistically significant difference. 
A

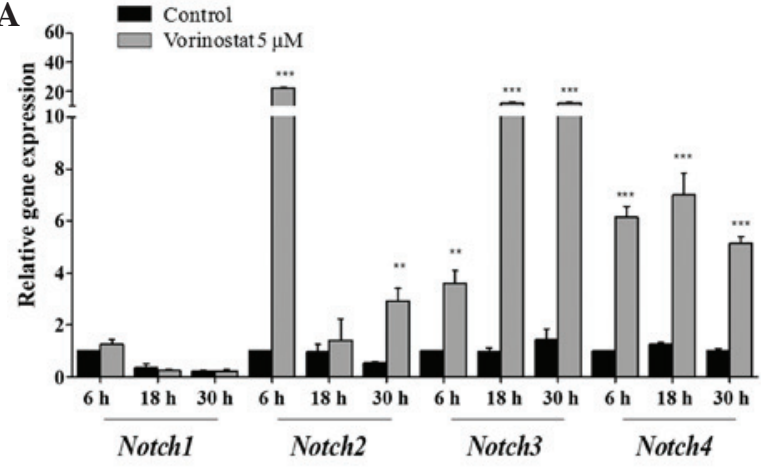

B

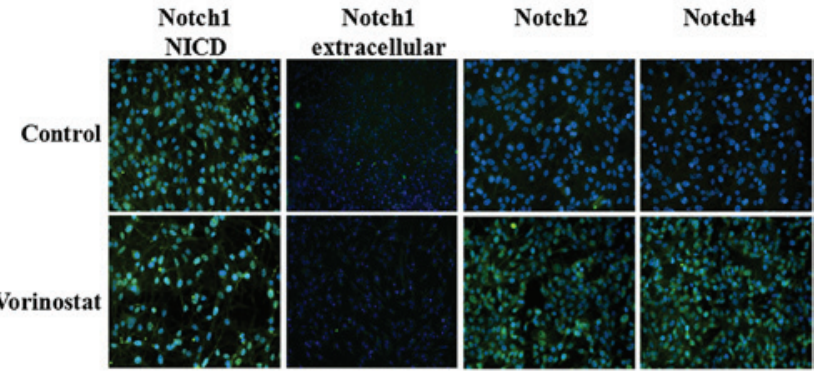

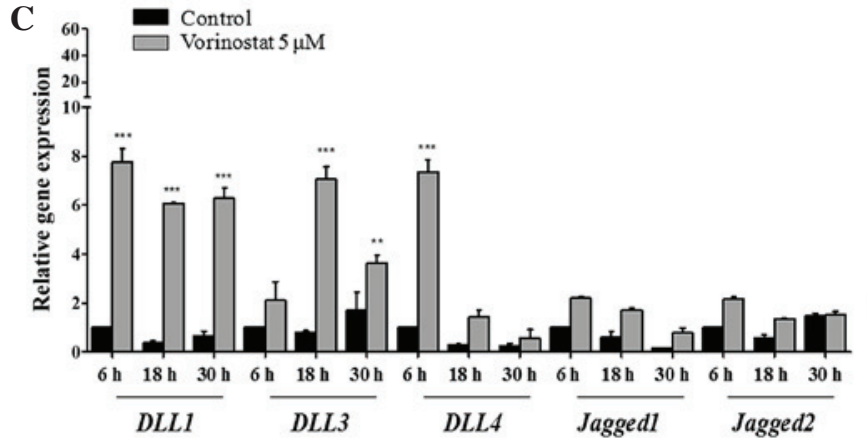

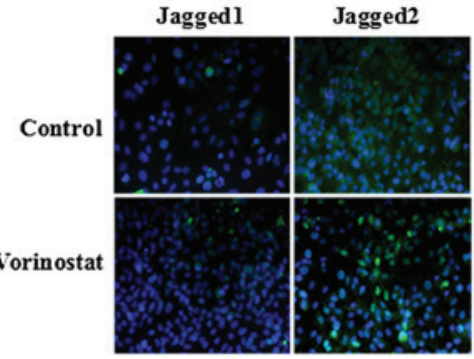

D
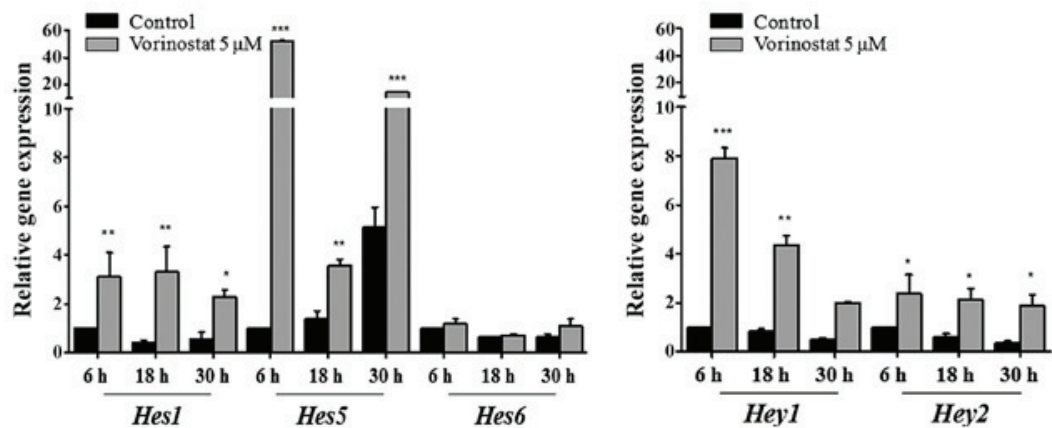

Figure 1. Vorinostat increases the mRNA expression of Notch receptors, Dll/Jagged ligands and Hey/Hes downstream target genes. Ovarian clear cell carcinoma ES2 cells were grown in the absence and presence of vorinostat $(5 \mu \mathrm{M})$ for 6,18 and $30 \mathrm{~h}$, following starvation, with medium supplemented with $1 \%$ fetal bovine serum. (A) Notch receptor expression levels in ES2 cells. RT-qPCR revealed that Notch2, Notch3 and Notch4 mRNA expression was increased following vorinostat exposure, whereas Notch1 was not differentially expressed. (B) Representative staining of Notch receptors by immunofluorescence. It was observed that there was an increase of Notch2 and Notch4 protein following exposure to vorinostat (magnification, x200). Nuclei are stained with 4',6-diamidino-2-phenylindole (blue) and Notch receptors with fluorescein isothiocyanate (green). (C) Notch ligand expression levels in ES2 cells. RT-qPCR indicated that D111, D113, D114 mRNA expression levels in cells treated with vorinostat were increased compared with cells without vorinostat treatment (magnification, x200). Jagged1 and Jagged2 mRNA expression levels were not significantly different. (D) Notch downstream target gene expression levels. RT-qPCR revealed that Hes1, Hes5, Heyl and Hey2 mRNA expression levels in cells treated with vorinostat were increased compared with cells without vorinostat treatment. Hes6 had a similar expression under all conditions. RT-qPCR was normalized to the hypoxanthine phosphoribosyltransferase gene. Data are presented as the mean \pm standard deviation of triplicate experiments. ${ }^{*} \mathrm{P}<0.05 ;{ }^{* * *} \mathrm{P}<0.01 ;{ }^{* * *} \mathrm{P} \leq 0.001$ vs. control cells. Dll, Delta-like; RT-qPCR, reverse transcription-quantitative polymerase chain reaction; Hes, hairy enhancer of split; Hey, Hes-related proteins.

\section{Results}

Effects of vorinostat treatment on Notch signaling in ovarian cancer cells. In order to investigate the effect of HDACi vorinostat on the Notch signaling pathway, OCCC ES2 and OSC OVCAR3 cell lines were exposed to vorinostat for various periods of time. The results demonstrated that vorinostat exposed OCCC ES2 cells express increased levels of Notch2 $(\mathrm{P}<0.010), 3$ and $4(\mathrm{P}<0.001)$ mRNAs, whereas Notch1 expression remained unchanged (Fig. 1A). Increased levels of Notch1 NICD in control and vorinostat exposed cells was observed using immunofluorescence. In Fig. 1B, immunofluorescence revealed that vorinostat exposure increased Notch2 and 4 protein expression but not extracellular Notch1. Concerning
Notch ligands in ES2 cells, vorinostat induced an increased expression of Dll1, Dll3 and Dll4 ( $\mathrm{P}<0.001)$, whereas Jagged1 and 2 levels remained the same compared with control cells (Fig. 1C). The significant increase in mRNA levels of Notch downstream genes Hesl $(\mathrm{P}<0.010)$, Hes5 $(\mathrm{P}<0.001)$, Heyl $(\mathrm{P}<0.010)$ and Hey2 $(\mathrm{P}<0.050)$, in cells exposed to vorinostat, confirmed the activation of Notch pathway in this cell line (Fig. 1D). In OVCAR3 cells, vorinostat also significantly increased Notch2, Notch3 and Notch4 $(\mathrm{P}<0.010)$, and slightly decreased Notch1 mRNA levels (Fig. 2A). Protein expression of Notch 2 and 4 was also higher in cells exposed to vorinostat; however, extracellular and NICD Notch1 levels were not increased (Fig. 2B). For Notch ligands, it was observed that there was a significant increase in Dl11 $(\mathrm{P}<0.010)$, Jagged1 and 
A

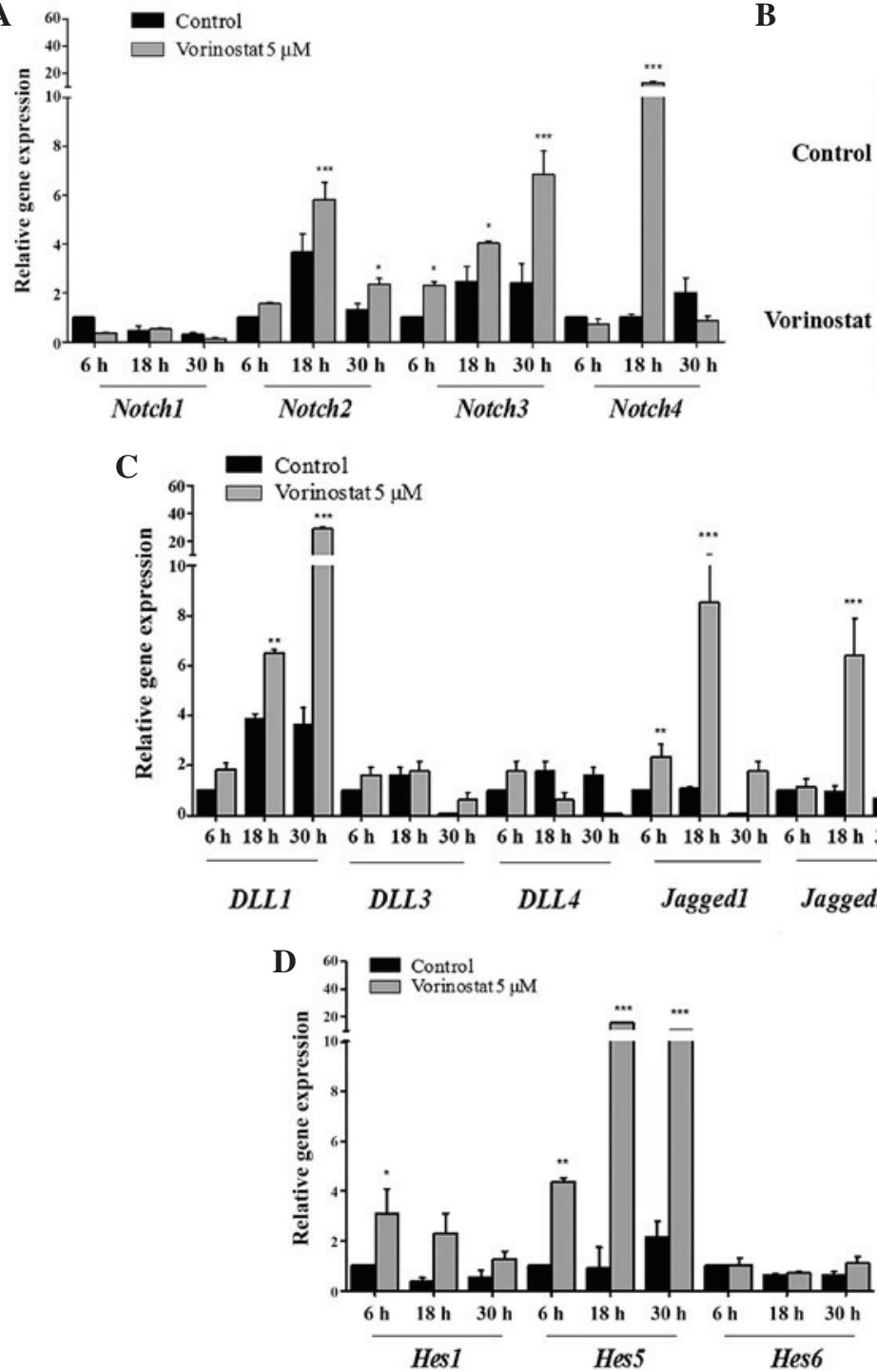

B
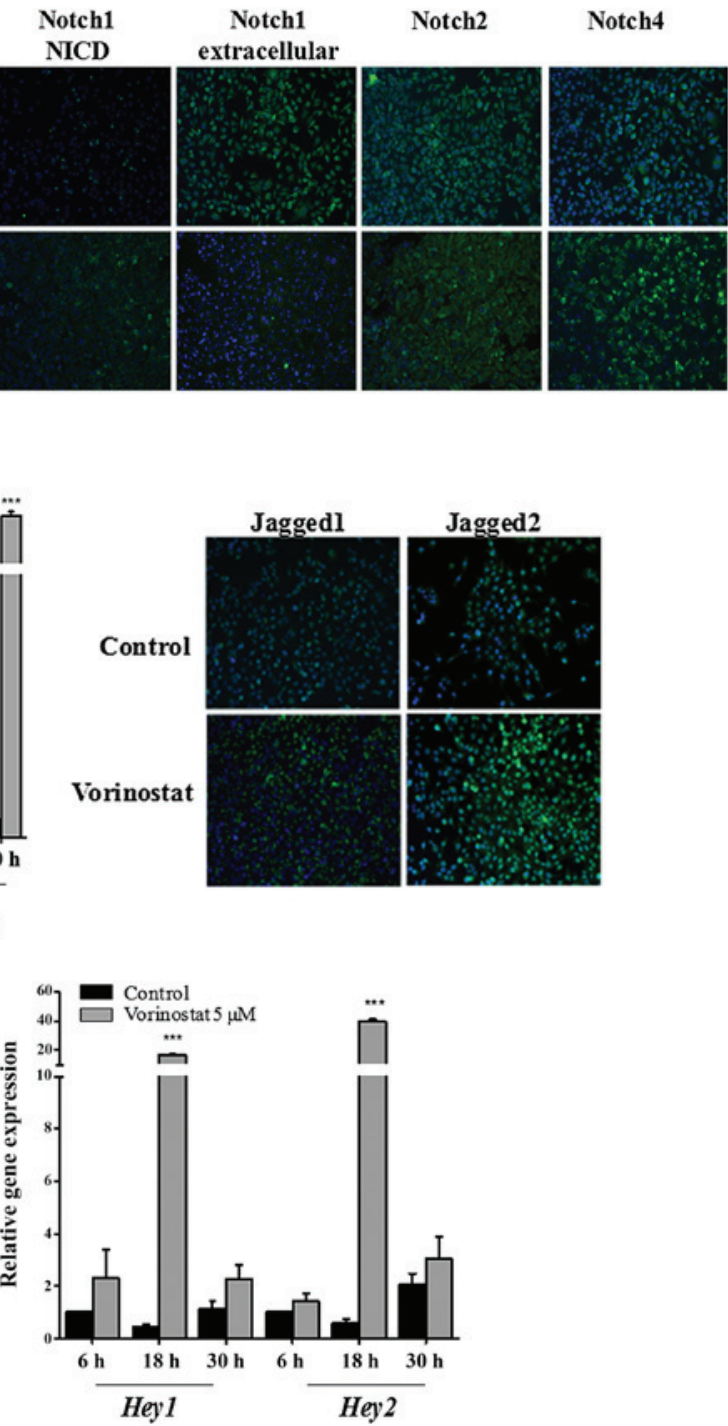

Figure 2. Vorinostat increases the mRNA expression of Notch receptors, ligands and downstream targets in ovarian serous carcinoma OVCAR3 cell line. The cells were grown in the absence and presence of vorinostat $(5 \mu \mathrm{M})$ for 6,18 and $30 \mathrm{~h}$, following starvation, with medium supplemented with $1 \%$ fetal bovine serum. (A) Notch receptor expression levels in OVCAR3 cells. RT-qPCR indicated that Notch2, Notch3 and Notch4 mRNA levels were increased following vorinostat exposure. (B) Representative staining of Notch receptors by immunofluorescence. Vorinostat slightly increased the protein expression of Notch receptors 2 and 4, and decreased Notch1 expression following exposure to vorinostat (magnification, x200). Nuclei are stained with 4',6-diamidino-2-phenylindole (blue) and Notch receptors with fluorescein isothiocyanate (green). (C) Notch ligand expression levels in OVCAR3 cell line. RT-qPCR revealed that D111, Jagged1 and Jagged2 mRNA expression levels in cells treated with vorinostat were increased compared with cells not treated with vorinostat (magnification, x200). D113 and D114 mRNA expression levels were not differentially expressed. (D) Notch downstream target gene expression levels. RT-qPCR revealed that Hes1, Hes5, Heyl and Hey2 mRNA expression levels in cells treated with vorinostat were increased compared with cells without vorinostat treatment. Hes 6 mRNA expression levels were not affected by vorinostat treatment. RT-qPCR was normalized to hypoxanthine phosphoribosyltransferase. Data are presented as the mean \pm standard deviation of triplicate experiments. ${ }^{*} \mathrm{P}<0.05 ;{ }^{* * *} \mathrm{P}<0.01 ;{ }^{* * *} \mathrm{P} \leq 0.001$ vs. control cells. D1l, Delta-like; RT-qPCR, reverse transpiration-quantitative polymerase chain reaction; Hes, hairy enhancer of split; Hey, Hes-related proteins.

Jagged2 $(\mathrm{P}<0.001)$ mRNA levels in OVCAR3 cells exposed to vorinostat (Fig. 2C), whereas Dll3 and 4 mRNA expression was not increased. Regarding Notch downstream targets, it was observed that vorinostat statistically increased Heyl, Hey2 $(\mathrm{P}<0.001)$, Hesl $(\mathrm{P}<0.050)$ and Hes5 $(\mathrm{P}<0.001)$, mRNA levels compared with control cells (Fig. 2D); however, Hes6 levels were not increased.

Overall, the present results reveal that vorinostat activated the Notch pathway in OCCC and OSC cell lines; however, this activation was through a different expression panel of Notch ligands in the two cell lines. In OCCC, the activation of Notch pathway appears to occur through Dl11, Dl12 and Dll3, whereas in OSC Dll1 and Jagged 1 and 2 ligand families appear to be involved. Nevertheless, the present results revealed that the activation of Notch pathway by vorinostat, in OCCC and OSC cell lines, culminated in the increased expression of the same downstream targets, Heyl, Hey2, Hes1 and Hes5.

\section{Discussion}

The Notch signaling pathway is an important cell signaling system that is activated in various types of cancer, including ovarian carcinoma $(5,30,31)$. Notch1 overexpression has been demonstrated in several studies to promote ovarian cancer cell proliferation (32-34), and this is primarily associated with increased levels of Notch1 NICD (33). In the literature, 

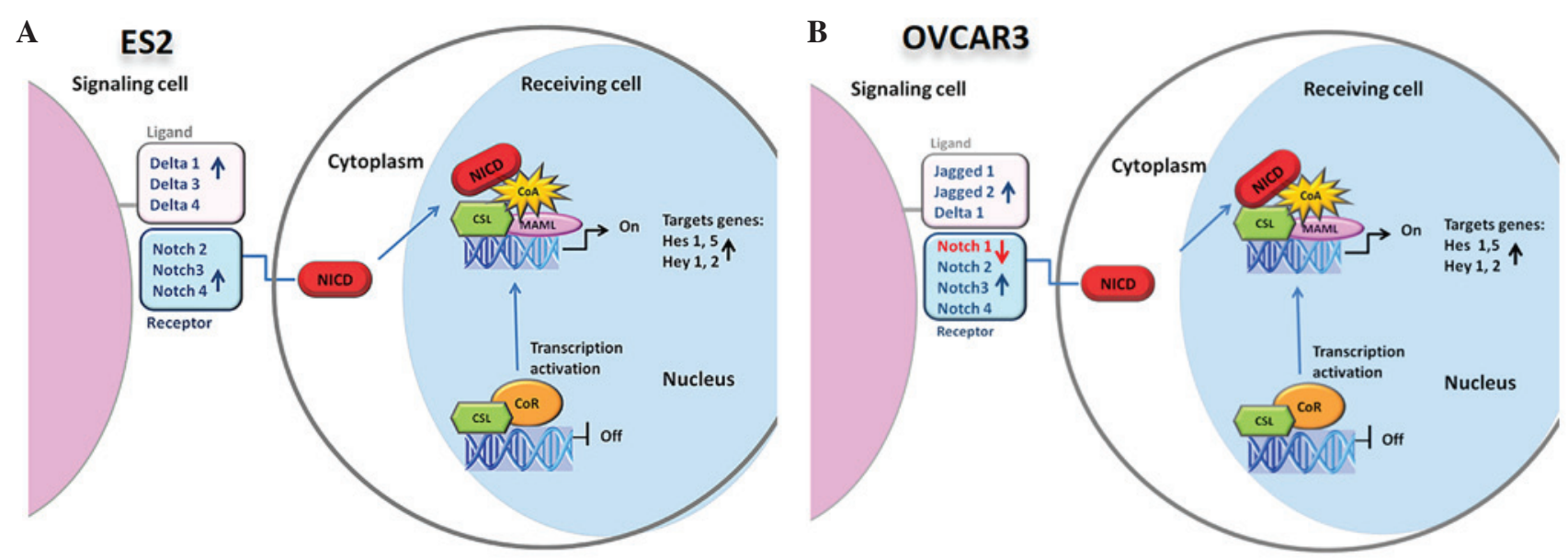

Figure 3. Schematic representation of the Notch pathway in ovarian (A) clear cell carcinoma ES2 and (B) serous carcinoma OVCAR3 cells following exposure to vorinostat. NICD, Notch intracellular domain; CSL, Suppressor of Hairless; CoR, corepressor; CoA, coactivator; MAML, mastermind-like proteins; Hes, hairy enhancer of split; Hey, Hes-related proteins.

Notch3 has also been demonstrated to be active in certain ovarian cancer cell lines and its overexpression was described in $20 \%$ of OSC $(35,36)$. In addition, Notch3 was revealed to increase following tumor chemotherapy, and its overexpression is associated with tumor aggressiveness and poor prognosis of patients (36).

The present study aimed to investigate the effect of vorinostat, an HDAC inhibitor, on the Notch signaling pathway in ovarian cancer cells. For this purpose, the present study used cell lines from two different histological types of EOC: OSC, the most prevalent and aggressive EOC type; and OCCC, which is a rare type of EOC that is highly resistant to chemotherapy (37).

No significant alteration in Notch1 mRNA levels following vorinostat exposure was identified in the two different ovarian cancer cell lines; however, high levels of cleaved Notch1 were detected in OCCC cell line (ES2). In addition, Notch3 was demonstrated to be upregulated following vorinostat exposure in the two cell lines. Furthermore, the present study revealed that vorinostat activates the Notch pathway through specific Notch ligands in OCCC and OSC cell lines; D111, 3 and 4 are activated in OCCC, while Dll1 and Jagged1 and 2 are activated in OSC. The present results are supported by a previous studies that demonstrated altered Notch signaling in OSC, due to increased expression of Jagged 1 and 2 ligands $(5,38)$.

The Hes and Hey gene families are the best characterized canonical Notch target genes, and the activation of Notch signaling upregulates their transcription (39); therefore, Hes and Hey mRNA expression may be considered as markers for Notch activation. The present study demonstrated that Hes and Hey Notch target genes are overexpressed in EOC cells following exposure to vorinostat. The present data reveals that vorinostat induces the overexpression of Hes and Hey Notch target genes in EOC cells, due to Notch signaling activation. In addition, the redundancy of Notch, Delta and Jagged elements expressed in EOC cell lines was shown (Fig. 3). Hence, no matter which panel of receptors or ligands is expressed on the cell membrane, the downstream target genes are always expressed in the presence of vorinostat. This upregulation can aid our understanding of the mechanism underlying the failure of vorinostat therapy in ovarian carcinoma.
In conclusion, the present findings illustrate the redundancy of Notch pathway in ovarian cancer, and suggest that disruption of histone acetylation may not be a useful therapeutic strategy in these carcinomas.

\section{References}

1. Reinhardt MJ: Gynecologic tumors. In: PET in Oncology. Dresel S (ed). Vol 170. Springer, Berlin, pp141-150, 2008.

2. Bast RC, Hennessy B and Mills GB: The biology of ovarian cancer: New opportunities for translation. Nat Rev Cancer 9: 415-428, 2009.

3. Siegel R, Naishadham D and Jemal A: Cancer statistics, 2013. CA Cancer J Clin 63: 11-30, 2013.

4. Smolle E, Taucher V, Pichler M, Petru E, Lax S and Haybaeck J: Targeting signaling pathways in epithelial ovarian cancer. Int J Mol Sci 14: 9536-9555, 2013.

5. Cancer Genome Atlas Research Network: Integrated genomic analyses of ovarian carcinoma. Nature 474: 609-615, 2011.

6. Del Carmen MG, Birrer M and Schorge JO: Clear cell carcinoma of the ovary: A review of the literature. Gynecol Oncol 126: 481-490, 2012.

7. Ayhan A, Mao TL, Seckin T, Wu CH, Guan B, Ogawa H, Futagami M, Mizukami H, Yokoyama Y, Kurman RJ and Shih IeM: Loss of ARID1A expression is an early molecular event in tumor progression from ovarian endometriotic cyst to clear cell and endometrioid carcinoma. Int J Gynecol Cancer 22: 1310-1305, 2012.

8. Guan B, Wang TL and Shih IM: ARID1A, a factor that promotes formation of SWI/SNF-mediated chromatin remodeling, is a tumor suppressor in gynecologic cancers. Cancer Res 71: 6718-6727, 2011.

9. Kotsopoulos IC, Papanikolaou A, Lambropoulos AF, Papazisis KT, Tsolakidis D, Touplikioti P and Tarlatzis BC: Serous ovarian cancer signaling pathways. Int J Gynecol Cancer 24: 410-417, 2014.

10. Jin KL, Pak JH, Park JY, Choi WH, Lee JY, Kim JH and Nam JH: Expression profile of histone deacetylases 1,2 and 3 in ovarian cancer tissues. J Gynecol Oncol 19: 185-190, 2008.

11. Ranganathan P, Weaver KL and Capobianco AJ: Notch signalling in solid tumours: A little bit of everything but not all the time. Nat Rev Cancer 11: 338-351, 2011.

12. Takebe N, Nguyen D and Yang SX: Targeting notch signaling pathway in cancer: Clinical development advances and challenges. Pharmacol Ther 141: 140-149, 2014.

13. Nickoloff BJ, Osborne BA and Miele L: Notch signaling as a therapeutic target in cancer: A new approach to the development of cell fate modifying agents. Oncogene 22: 6598-6608, 2003.

14. Aster JC and Blacklow SC: Targeting the Notch pathway: Twists and turns on the road to rational therapeutics. J Clin Oncol 30: 2418-2420, 2012.

15. Li JL and Harris AL: Notch signaling from tumor cells: A new mechanism of angiogenesis. Cancer Cell 8: 1-3, 2005. 
16. Dufraine J, Funahashi Y and Kitajewski J: Notch signaling regulates tumor angiogenesis by diverse mechanisms. Oncogene 27: 5132-5137, 2008.

17. Fleming RJ, Purcell K and Artavanis-Tsakonas S: The NOTCH receptor and its ligands. Trends Cell Biol 7: 437-441, 1997.

18. Lendahl U: A growing family of Notch ligands. Bioessays 20: 103-107, 1998.

19. Gray GE, Mann RS, Mitsiadis E, Henrique D, Carcangiu ML, Banks A, Leiman J, Ward D, Ish-Horowitz D and Artavanis-Tsakonas S: Human ligands of the Notch receptor. Am J Pathol 154: 785-794, 1999.

20. Yamaguchi E, Chiba S, Kumano K, Kunisato A, Takahashi T, Takahashi T and Hirai H: Expression of Notch ligands, Jagged1, 2 and Delta1 in antigen presenting cells in mice. Immunol Lett 81 : 59-64, 2002.

21. Kao HY, Ordentlich P, Koyano-Nakagawa N, Tang Z, Downes M, Kintner CR, Evans RM and Kadesch T: A histone deacetylase corepressor complex regulates the Notch signal transduction pathway. Genes Dev 12: 2269-2277, 1998.

22. Ronchini $C$ and Capobianco AJ: Induction of cyclin D1 transcription and CDK2 activity by Notch (ic): Implication for cell cycle disruption in transformation by Notch (ic). Mol Cell Biol 21: 5925-5934, 2001

23. Weng AP, Millholland JM, Yashiro-Ohtani Y, Arcangeli ML, Lau A, Wai C, Del Bianco C, Rodriguez CG, Sai H, Tobias J, et al $\mathrm{c}-\mathrm{Myc}$ is an important direct target of Notch1 in T-cell acute lymphoblastic leukemia/lymphoma. Genes Dev 20: 2096-2109, 2006.

24. Hirose H, Ishii H, Mimori K, Ohta D, Ohkuma M, Tsujii H, Saito T, Sekimoto M, Doki Y and Mori M: Notch pathway as candidate therapeutic target in Her2/Neu/ErbB2 receptor-negative breast tumors. Oncol Rep 23: 35-43, 2010.

25. Takai $\mathrm{N}$ and Narahara H: Histone deacetylase inhibitor therapy in epithelial ovarian cancer. J Oncol 2010: 458431, 2010.

26. Bolden JE, Peart MJ and Johnstone RW: Anticancer activities of histone deacetylase inhibitors. Nat Rev Drug Discov 5: 769-784, 2006.

27. Richon VM: Cancer biology: mechanism of antitumour action of vorinostat (suberoylanilide hydroxamic acid), a novel histone deacetylase inhibitor. Br J Cancer 95 (Suppl 1): S2-S6, 2006.
28. Cooper AL, Greenberg VL, Lancaster PS, Van Nagell JR Jr, Zimmer SG and Modesitt SC: In vitro and in vivo histone deacetylase inhibitor therapy with suberoylanilide hydroxamic acid (SAHA) and paclitaxel in ovarian cancer. Gynecol Oncol 104: 596-601, 2007.

29. Livak KJ and Schmittgen TD: Analysis of relative gene expression data using real-time quantitative PCR and the 2(-Delta Delta C(T)) Method. Methods 25: 402-408, 2001.

30. Bolós V, Grego-Bessa J and de la Pompa JL: Notch signaling in development and cancer. Endocr Rev 28: 339-363, 2007.

31. McAuliffe SM, Morgan SL, Wyant GA, Tran LT, Muto KW, Chen YS, Chin KT, Partridge JC, Poole BB, Cheng KH, et al: Targeting Notch, a key pathway for ovarian cancer stem cells, sensitizes tumors to platinum therapy. Proc Natl Acad Sci USA 109: E2939-E2948, 2012.

32. Rose SL, Kunnimalaiyaan M, Drenzek J and Seiler N: Notch 1 signaling is active in ovarian cancer. Gynecol Oncol 117: 130-133, 2010.

33. Hopfer O, Zwahlen D, Fey MF and Aebi S: The Notch pathway in ovarian carcinomas and adenomas. Br J Cancer 93: 709-718, 2005.

34. Capaccione KM and Pine SR: The Notch signaling pathway as a mediator of tumor survival. Carcinogenesis 34: 1420-1430, 2013.

35. Park JT, Li M, Nakayama K, Mao TL, Davidson B, Zhang Z, Kurman RJ, Eberhart CG, Shih IeM and Wang TL: Notch3 gene amplification in ovarian cancer. Cancer Res 66: 6312-6387, 2006.

36. Park JT, Chen X, Tropè CG, Davidson B, Shih IM and Wang TL: Notch3 overexpression is related to the recurrence of ovarian cancer and confers resistance to carboplatin. Am J Pathol 177: 1087-1094, 2010

37. Tan DSP, Miller RE and Kaye SB: New perspectives on molecular targeted therapy in ovarian clear cell carcinoma. $\mathrm{Br}$ J Cancer 108: 1553-1559, 2013.

38. Jung SG, Kwon YD, Song JA, Back MJ, Lee SY, Lee C, Hwang YY and An HJ: Prognostic significance of Notch 3 gene expression in ovarian serous carcinoma. Cancer Sci 101: 1977-1983, 2010.

39. Iso T, Kedes L and Hamamori Y: HES and HERP families: Multiple effectors of the Notch signaling pathway. J Cell Physiol 194: 237-255, 2003. 